\title{
Delays for Utilizing Institutional Delivery and Associated Factors Among Mothers Attending Public Health Facility in Hadiya Zone, Southern Ethiopia
}

\author{
Alemu Lire ${ }^{1}$, Abera Beyamo ${ }^{2,}$, , Degefa Tadele ${ }^{1}$, Wolde Facha ${ }^{2}$ \\ ${ }^{1}$ Hadiya Zone Health Department, Hosana, Ethiopia \\ ${ }^{2}$ Public Health Department, College of Health Sciences, Wolaita Sodo University, Wolaita Sodo, Ethiopia \\ Email address: \\ bdereje05@gmail.com (A. Beyamo),dilolombamo@gmail.com (A. Lire), degefa04@gmail.com (D. Tadele), \\ woldiefacha@gmail.com (W. Facha) \\ ${ }^{*}$ Corresponding author
}

To cite this article:

Alemu Lire, Abera Beyamo, Degefa Tadele, Wolde Facha. Delays for Utilizing Institutional Delivery and Associated Factors Among Mothers Attending Public Health Facility in Hadiya Zone, Southern Ethiopia. Science Journal of Public Health.

Vol. 5, No. 6, 2017, pp. 419-427. doi: 10.11648/j.sjph.20170506.13

Received: July 29, 2017; Accepted: August 21, 2017; Published: September 28, 2017

\begin{abstract}
Labour and delivery are the shortest and most critical period during pregnancy and childbirth. The major factors that contribute to maternal death in developing countries are: delay in deciding to seek care, identifying and reaching medical facility, and receiving adequate and appropriate treatment. The study aimed to determine delays in institutional delivery and associated factors among mothers attending public health facilities, southern Ethiopia. Facility based Cross sectional study was employed on 397 labouring mothers attending Negist Elene Mohamed memorial general hospital. The sample size was determined by using single population proportion formula and the data were collected consecutively until the required sample sizes achieved. Descriptive data analysis was conducted to identify summary values and multiple logistic regressions were performed to identify independent predictors for the delays. All the analysis was done using SPSS for windows version 16.0. A total of 384 labouring mothers were participated in this study. The proportion of mothers who exhibited the first delay was 154(40.1\%). Maternal unemployment [AOR, 2.5; 95\% CI, 1.118, 5.441]; husband educational status [AOR, 2.3; 95\% CI, $1.204,4.408$ ]; and antenatal care visit [AOR, $0.4 ; 95 \%$ CI, 0.277, 0.713] were the independent predictors of the first delay. Mothers who experienced the second delay were 114(29.7\%). For this delay, distance [AOR, 14; CI, 7.895, 26.558]; uneducated mothers [AOR, 3; 95\% CI, 1.397, 6.711]; and means of transportation [AOR, 0.6; 95\% CI, 0.314, 0.995] were determinants. Mothers who experienced the $3^{\text {rd }}$ delay were $125(32.6 \%)$. The main predictors were multiple referral levels [AOR, 0.2; 95\% CI, 0.068, 0.347]; absence of care provider [AOR, 1.7; 95\% CI, 1.060, 2.697]; and lengthy admission process [AOR, 2.2; $95 \% \mathrm{CI}, 1.279,3.776]$. The very high percentage of each delay in this study suggests low utilization of emergency obstetric care among labouring mothers.
\end{abstract}

Keywords: Delays, Emergency Obstetric Care, Ethiopia

\section{Introduction}

Delay for utilizing institutional delivery refers to the time interval from deciding to seek emergency care to start in receiving of first healthcare. First delay refers to the time interval from the first onset of labour to decision to seek emergency obstetric care. It is one of the contributing factors for high maternal mortality in developing countries. Second delay refers to delay to go to health facility after the decision has been made to seek emergency obstetric care. It is a delay in physically reaching to the nearby care facility within an hour after deciding to seek healthcare. Third delay refers to receive appropriate care once present at the health facility within the first five minutes of arrival. Delays are the major contributing factors for high maternal mortality $[\mathrm{MM}]$ in developing countries $[1,2]$. 
Around $15 \%$ of all pregnant women develop a possibly life-threatening complication that calls for skilled delivery care, and some will require a major obstetrical intervention to survive. About 1,000 women die from pregnancy/childbirth related complications around the world every day and more than half a million women die each year; of these, $99 \%$ occur in low-resource countries. Improving maternal health is one of the eight Millennium Development Goals (MDGs) adopted by the international community in 2000 and designed to achieve a 75\% reduction in MM between 1990 and $2015[3,4,5]$.

Various models have been developed to strengthen the coverage and quality of maternal health services. One of the most widely applied models in maternal health programming today is the three delays model which promotes the presence of a skilled birth attendant who is linked to a functioning health system. Thaddeus and Maine (1994) has provided the safe motherhood community with an explanatory model of MM that identifies the key factors leading to maternal death. This explanatory model categorizes delays into three types: the delays of the decision to seek health care, the ability to get access to a health facility (HF), and receiving medical care timely and appropriately once they reached facility $[1,6]$.

Skilled birth attendants play an important role in reducing maternal and neonatal mortality because they provide timely obstetric and newborn care for lifethreatening complications [7].

The occurrence of all obstetric complications may not be preventable but almost all are treatable before resulting in serious maternal and perinatal morbidity and mortality $[8,9]$.

Institutional delivery is staggering low in our country. According to 2014 Ethiopian mini demographic and health survey (EDHS), only $14.9 \%$ of total pregnant women were delivered in HFs [12]. This represented an improvement from $6.2 \%$ in 2000 and $10 \%$ in 2011. But, rate is in the lowest bound by sub-Saharan Africa standard. As projected to increase at a low pace of 5.6\% per annum for the MDG target year of 2015, it is very disappointed change [10].

Labour and delivery are the shortest and most critical period during pregnancy and childbirth because most maternal deaths arise from complications during delivery. Even with the best possible antenatal care, it is established that delivery could be complicated; therefore, timely referrals and access to appropriate health care had a great impact on reduction to MMs and disabilities [11].

Even if number of attempts vis-à-vis to delays and associated factors has been made to show how these factors affect EmOC, it is moving unsteadily. Specifically, the problem of maternal health service delays in skilled birth attendance at the proposed study area is still not responded question and no research addresses to this problem. Thus, this study was conducted, in order to determine proportion of delays for utilizing institutional delivery and associated factors among mothers attending public health facility in Hadiya zone, southern Ethiopia.

\section{Methods}

\subsection{Study Design and Area}

Facility based cross-sectional study was conducted among mothers attending public health facility in Hadiya zone, southern Ethiopia, from March 08 to April 08, 2015 in Negist Elene Mohamed memorial general public hospital. The zone is divided into 10 Woredas and 1 town administration with a total of 305 Rural and 24 Urban Kebeles. Based on population projection, the total population of the zone in $2013 / 14$ was estimated to be $1,547,846$. From the total population, $49.47 \%$ male and $50.53 \%$ percent were females. Reproductive age women accounted for about $23.3 \%$ percent. The zone has one zonal hospital, 63 public health centers, 305 health posts, and 81 private clinics. Negest Elene memorial general hospital is the only tertiary (zonal) hospital found in the Hossana town, capital city of Hadiya zone. It serves for over 1.5 million people residing in urban and rural parts of the zone. Mothers who were presented in person at study area for institutional delivery service during the data collection time and giving consent to participate were included in the study.

\subsection{Study Population and Sampling}

The sample size was calculated using a single population proportion formula; considering $37.8 \%$ proportion of EmOC delay of the study done in Ethiopia [12], 5\% margin of error and $10 \%$ estimated non-response rate, the calculated sample size required for the study was 397 labouring mothers. The selection of labouring mothers for the interview was based on consecutive sampling until the required sample size was obtained. Ethical clearance was obtained from Research and Graduate Studies College of Health Sciences Ethical Review Board of Jimma University. Formal letters from the Ethical review committee of Jimma University to Zonal Health Department, then letter of permission was produced from administrative bodies of the Zone to the Hospital administration. Finally written consent was obtained from each study participants before making interview and confidentiality was secured.

\subsection{Data Collection Instrument}

Data was collected by using originally prepared and pretested interviewer administered structured questionnaire that was in English and translated into local language Hadiyisa and Amharic and then back translated into English by two independent experts to check consistency. The tools were mainly addressing on: socio-demographic characteristics, obstetric history, delays, and knowledge about maternal healthcare services.

\subsection{Data Collectors}

Four diploma nurses for data collection and two BSC nurses for supervision were recruited. One day training on how to fill the questionnaire, request the consent, assure confidentiality of information of the study participants was 
given to data collectors and supervisors to ensure the quality of the field operation by principal investigator. During data collection, the supervisors had supervised the data collection process in daily base and perform quality checks. The instrument was pre tested on $5 \%$ of the sample to check reliability of the tool. Based on the findings and feedback obtained from the pre-testing process, modification was done on the questionnaire. The pre-tested data was not included in the main data analysis.

\subsection{Data Processing}

All collected data from questionnaire was checked manually before entry to software. Then, the data was entered in to a computer using EpiData version 3.1 software. The software was created based on data type and size, categories, validating permitted values and ranges, and codes to missing value. Corrections were made according to the original data. Finally, the data was exported to SPSS version 16 software for analysis.

\subsection{Data Analysis}

The data was analyzed by using SPSS version 16 software. Descriptive analysis was carried out for each of the variables to check frequency, distribution and missing value. Bivariate analysis was employed to check crude association between delays in emergency obstetric care and independent variables. Chi-square test was conducted to see variables fulfilling assumption. Variable with $p$ value $<0.25$ on bivariate analysis was entered to multivariable logistic regression to identify the factors that affect delays in emergency obstetric care. Binary logistic regression test was used to assess association between independent variables and delays in emergency obstetric care. Odds ratio and corresponding $95 \%$ confidence intervals was used to quantify the degrees of association between independent variable and delays in emergency obstetric care. Results with p-value $\leq 0.05$ were considered as being statistically significant and the rest was refuted. Multicollinearity among independently associated variables was checked by multicollinearity diagnostic test VIF in linear regression and none was collinear.

\section{Results}

\subsection{Socio-demographic Characteristics of the Respondents}

In this study 384 of the study subjects were interviewed resulting in a response rate of $96.7 \%$. Of these, $220(57.3 \%)$ were rural dweller, $301(78.4 \%)$ were found in the age group of 20-34 years, 274(71.4\%) were Hadiya, 236(61.5\%) protestant and 366(95.3\%) were married see (table 1).

Table 1. Socioeconomic, demographic and behavioral factors associated with institutional delivery delays among labouring mothers, 2015.

\begin{tabular}{|c|c|c|c|}
\hline Variable & & Frequency & Percent (\%) \\
\hline \multirow{2}{*}{ Residence } & Urban & 164 & 42.7 \\
\hline & Rural & 220 & 57.3 \\
\hline \multirow{3}{*}{ Age group } & $<20$ & 37 & 9.6 \\
\hline & $20-34$ & 301 & 78.4 \\
\hline & $\geq 35$ & 46 & 12.0 \\
\hline \multirow{6}{*}{ Ethnicity } & Hadiya & 274 & 71.4 \\
\hline & Kambata & 45 & 11.7 \\
\hline & Silte & 30 & 7.8 \\
\hline & Amhara & 10 & 2.6 \\
\hline & Gurage & 19 & 4.9 \\
\hline & Others & 6 & 1.6 \\
\hline \multirow{4}{*}{ Religion } & Protestant & 274 & 61.5 \\
\hline & Orthodox & 75 & 19.5 \\
\hline & Muslim & 55 & 14.3 \\
\hline & Catholic & 18 & 4.7 \\
\hline \multirow[t]{2}{*}{ Marital status } & Single & 10 & 2.6 \\
\hline & Divorced & 8 & 2.1 \\
\hline \multirow{5}{*}{ Education of the mothers } & Can't read \& write & 85 & 22.1 \\
\hline & Can read \& write & 88 & 22.9 \\
\hline & Formal education & 47 & 12.2 \\
\hline & Primary education & 59 & 15.4 \\
\hline & Secondary education & 105 & 27.3 \\
\hline \multirow{5}{*}{ Education of husband } & Can't read \& write & 51 & 13.3 \\
\hline & Can read \& write & 71 & 18.5 \\
\hline & Formal education & 39 & 10.2 \\
\hline & Primary education & 58 & 15.1 \\
\hline & Secondary education & 165 & 43.0 \\
\hline \multirow{2}{*}{ Husband occupation } & Employed & 129 & 33.6 \\
\hline & Unemployed & 255 & 66.4 \\
\hline Monthly income & $\leq 1000 \mathrm{ETB}$ & 210 & 54.7 \\
\hline
\end{tabular}




\begin{tabular}{llll}
\hline Variable & & Frequency & Percent (\%) \\
\hline \multirow{3}{*}{ Decision maker for EmOC } & Family & 269 & 70.1 \\
& Mother & 53 & 13.8 \\
Parity & Husband & 62 & 16.1 \\
& Primi-para & 240 & 62.5 \\
Delay in decision & Multipara & 144 & 37.5 \\
& Not delayed & 230 & 59.9 \\
Number of ANC visit & Delayed & 154 & 40.1 \\
& $<4$ visit & 262 & 68.2 \\
Knowledge & $\geq 4$ visits & 122 & 31.8 \\
& Poor & 203 & 52.9 \\
Delay in reaching & Good & 181 & 47.1 \\
& Not delayed & 270 & 70.3 \\
Delay in receiving care & Delayed & 114 & 29.7 \\
& Not delayed & 259 & 67.4 \\
Type of delivery & Delayed & 125 & 32.6 \\
\hline
\end{tabular}

Note: $\mathrm{SVD}=$ spontaneous vaginal delivery, $\mathrm{NSVD}=$ non spontaneous vaginal delivery

\subsection{The Prevalence of Delays}

\subsubsection{The Delay in Decision/Maternal Delay}

The finding of this study showed that 154(40.1\%) of the study subjects didn't make decision to seek emergency obstetric care from health facilities within one hour of the commencement of labour. The reasons for mothers to delay in decision making to seek care was previous normal pregnancy outcome in $47(41.2 \%)$ of the mothers, labour starting at night in $35(30.7 \%)$ of the mothers, no money for transportation 12(10.5), no care for children remain at home 5(4.4)and 158(91.3\%) mothers for why delay in decision making was when the labouring mother carried on by stretcher (community transport) she ought to cover all the cost (food and drinking) of the accompanied individuals her to health facility.

\subsubsection{The Delay in Reaching to Healthcare Facility}

The findings in this study revealed that $114(29.7 \%)$ of mothers unable to arrive at the place of delivery for getting emergency obstetric services within one hour of travelling on foot/local transportation. The mean walking time took one and half hours with a SD \pm 0.457 to reach the healthcare facilities.

\subsubsection{The Third Delay in Receiving Care}

At health facilities, out of 384 study subjects 125 (32.6\%) mothers did not get an emergency obstetric care within the first five minutes of arrival to health facility. The mean delay time was two hours with $\mathrm{SD} \pm 0.469$.

\subsection{Factors Associated with Three Delays}

\subsubsection{Factors for First Delay}

In binary logistic regression analysis: mother's age, place of residence, mothers and husband's occupation, educational status of spouse, monthly income, parity, decision maker, knowledge on institutional delivery importance, and ANC follow up were found to be significantly associated with the first delay at a $p$-value $\leq 0.25$; to control the confounding effect of variables and identify the independent predictors a multiple logistic regression analysis was done for those found to have statistically significant explanatory variables during the binary analysis.

In doing so, mother's delay in decision to seek emergency obstetric care was 2.5 times higher among unemployed mother [AOR, 2.5; 95\% CI, 1.118, 5.441] than employed ones it was also found that mothers with illiterate husbands were at higher risk of delaying in seeking emergency obstetric care than their counterparts [AOR, 2.3; 95\% CI, 1.204, 4.408]; mothers who attended greater than or equal to four ANC visits were [AOR, $0.4 ; 95 \% \mathrm{CI}, 0.277,0.713] 60 \%$ less likely to delay than attended less than four visits. (See Table 2)

Table 2. Multiple logistic regression analysis result on associated factors of first delays in decision to seek EmOC services among labouring mothers in Hadiya zone, $2015(n=384)$.

\begin{tabular}{|c|c|c|c|c|c|}
\hline \multirow{2}{*}{ Variable } & & \multicolumn{4}{|l|}{ First delay } \\
\hline & & Delayed & Not delayed & COR $(95 \%$ CI $)$ & AOR (95\% CI) \\
\hline \multirow{2}{*}{ Residence } & Urban & $58(35.4)$ & $106(64.6)$ & 1 & 1 \\
\hline & Rural & $96(43.6)$ & $124(56.4)$ & $1.4(0.933,2.146)$ & $1.0(0.707,1.791)$ \\
\hline \multirow{3}{*}{ Age group } & $15-20$ & $8(21.6 \%)$ & $29(78.4 \%)$ & $0.4(0.165,0.843)$ & $0.6(0.200,1.658)$ \\
\hline & $21-34$ & $128(42.5 \%)$ & $173(57.5 \%)$ & 1 & 1 \\
\hline & $\geq 35$ & $18(39.1 \%)$ & $28(60.9 \%)$ & $0.9(0.461,1.639)$ & $1.7(0.831,3.410)$ \\
\hline \multirow{2}{*}{ Education of the mothers } & Illiterate & $105(47.7)$ & $115(52.3)$ & $2.1(1.399,3.282)$ & $0.8(0.386,1.458)$ \\
\hline & Literate & $49(29.9)$ & $115(70.1)$ & 1 & 1 \\
\hline \multirow{2}{*}{ Education of husband } & Illiterate & $86(53.4)$ & $75(46.6)$ & $2.6(1.716,3.982)$ & $2.3(1.204,4.408)^{*}$ \\
\hline & Literate & $68(30.5)$ & $155(69.5)$ & 1 & 1 \\
\hline
\end{tabular}




\begin{tabular}{llllll}
\hline \multirow{2}{*}{ Variable } & & First delay & & \\
\cline { 3 - 5 } & & Delayed & Not delayed & COR (95\% CI) & AOR (95\% CI) \\
\hline \multirow{2}{*}{ Husband occupation } & Employed & $35(27.1)$ & $94(72.9)$ & 1 & 1 \\
& Unemployed & $119(46.7)$ & $136(53.3)$ & $2.4(1.484,3.721)$ & $1.1(0.530,2.222)$ \\
Mothers occupation & Employed & $30(27 \%)$ & $81(73 \%)$ & 1 & 1 \\
& Unemployed & $124(45.4)$ & $149(54.6)$ & $3.4(1.880,6.141)$ & $2.5(1.118,5.441)^{*}$ \\
Monthly income & $\geq 2000$ ETB & $48(32.4 \%)$ & $100(67.6 \%)$ & 1 & 1 \\
& $1001-1999$ ETB & $11(42.3)$ & $15(57.7)$ & $1.5(0.653,3.577)$ & $1.2(0.482,3.230)$ \\
& $\leq 1000$ ETB & $95(45.2)$ & $115(54.8)$ & $1.7(1.110,2.668)$ & $0.8(0.437,1.369)$ \\
Decision maker for EmOC & Family & $106(39.4)$ & $163(60.6)$ & 1 & 1 \\
& Mother & $17(32.1)$ & $36(67.9)$ & $0.7(0.388,1.359)$ & $0.8(0.388,1.507)$ \\
Knowledge & Husband & $31(50.0)$ & $31(50)$ & $1.5(0.883,2.678)$ & $1.4(0.760,2.602)$ \\
& poor & $108(43.9)$ & $138(56.1)$ & $1.6(1.014,2.417)$ & $0.8(0.480,1.306)$ \\
Number of ANC visit & Good & $46(33.3)$ & $92(66.7)$ & 1 & 1 \\
& $<4$ visit & $84(32.1)$ & $178(67.9)$ & 1 & 1 \\
& $\geq 4$ visits & $70(57.4)$ & $52(42.6)$ & $0.4(0.225,0.546)$ & $0.4(0.277,0.713)^{* *}$ \\
\hline
\end{tabular}

\subsubsection{Factors for Second Delay}

In binary logistic regression analysis: educational status of the mother and husband, monthly income, distance from health facility, means of transportation (ambulance against others), and road accessibility were found to be candidate variables for multivariable logistic regression with the second delay at a $p$-value of $\leq 0.25$; and in multivariable logistic regression: distance, means of transportation, and educational status of the mother were independently associated at a $p$ value of $<0.05$. Accordingly, mothers those travelling beyond 5 kilometres from health facilities were 14 times more likely to delay in reaching to healthcare facility [AOR, 14; CI, 7.895, 26.558] compared to those living within five kilometres; educational status of the mother was also found to predict the second delay putting not-educated mothers at 3 times higher risk [AOR, 3.0; 95\% CI, 1.397, 6.711]; and mothers who used other means of transportation [AOR, 0.6; $95 \%$ CI, $0.314,0.995] 40 \%$ more likely to delays than those used ambulance.(See Table 3 below)

Table 3. Factors associated with delays in reaching to health facility, $2015(n=384)$.

\begin{tabular}{llllll}
\hline \multirow{2}{*}{ Variable } & \multicolumn{4}{l}{ Delay in getting service } \\
\cline { 2 - 5 } & & Delayed & Not delayed & COR 95\% CI & AOR 95\% CI \\
\hline \multirow{2}{*}{ Education of the mothers } & Illiterate & $88(40.0)$ & $132(60.0)$ & $3.5(2.150,5.824)$ & $3.1(1.397,6.711)^{*}$ \\
& Literate & $26(15.9)$ & $138(84.1)$ & 1 & 1 \\
\multirow{2}{*}{ Education of husband } & Illiterate & $70(43.5)$ & $91(56.5)$ & $3.1(1.988,4.926)$ & $1.4(0.693,2.940)$ \\
& Literate & $44(19.7)$ & $179(80.3)$ & 1 & 1 \\
Monthly income & $\geq 2000$ ETB & $26(17.6)$ & $122(82.4)$ & 1 & 1 \\
& $1001-1999$ ETB & $4(15.4)$ & $22(84.6)$ & $0.8(0.271,2.685)$ & $0.6(0.148,2.649)$ \\
Distance & $\leq 1000$ ETB & $84(40.0)$ & $126(60.0)$ & $3.1(1.887,5.186$ & $1.4(0.743,2.749)$ \\
& $\geq 5 \mathrm{~km}$ & $93(58.5)$ & $66(41.5)$ & $13.7(7.906,23.699)$ & $14(7.895,26.558)^{* *}$ \\
Means of transportation & $<5 \mathrm{~km}$ & $21(9.3)$ & $204(90.7)$ & 1 & 1 \\
& Ambulance & $57(39.6)$ & $87(60.4)$ & 1 & 1 \\
Road accessibility & Others & $57(23.8)$ & $183(76.2)$ & $0.5(0.304,0.743)$ & $0.6(0.314,0.995) *$ \\
& Yes & $60(25.3)$ & $177(74.7)$ & 1 & 1 \\
& No & $54(36.7)$ & $93(63.3)$ & $1.7(1.097,2.673)$ & $1.4(0.844,2.640)$ \\
\hline
\end{tabular}

Note: $1=$ reference, $*=$ p-value $<0.05$, and $* *=$-value $\leq 0.001$

\subsubsection{Factors for Third Delay}

To identify predictors in the third delay, the same statistical analysis was tracked as applied in first delay. Initially, each independent variable such as requesting multiple referrals, absence of care provider, lengthy admission process, and type of delivery was regressed against dependent variable. Except the type of delivery other variables were established statistically significant association with the third phase delay at a $p$-value of $<0.05$. The findings in this study showed that mothers who experienced multiple referral levels were [AOR, 0.2; 95\% CI, 0.068, 0.347] more likely to delay than utilized institutional delivery at first contact of visit; it was also found that mothers who didn't get appropriate care provider and faced lengthy admission process were $1.7 \& 2.2$ times at higher risk of delaying in receiving EmOC than their counter parts [AOR, 1.7; 95\% CI, 1.060, 2.697] and [AOR, $2.2 ; 95 \%$ CI, $1.279,3.776]$, respectively. (See table 4) 
Table 4. Delays in getting care (3rd delay) at health facility level and associated factors, Hadiya Zone NEM general Hospital, 2015 ( $n=384)$.

\begin{tabular}{llllll}
\hline \multirow{2}{*}{ Variable } & \multicolumn{4}{l}{ Delay in getting services } & \\
\cline { 2 - 5 } & \multicolumn{1}{l}{ Delayed } & Not delayed & COR(95\% CI) & AOR (95\% CI) \\
\hline \multirow{2}{*}{ Multiple referral } & Yes & $7(8.4)$ & $76(91.6)$ & $0.2(0.064,0.320)$ & $0.2(0.068,0.347)^{* *}$ \\
& No & $118(39.2)$ & $183(60.8)$ & 1 & 1 \\
Absence of care provider & Yes & $54(40.3)$ & $80(59.7)$ & $1.7(1.095,2.645)$ & $1.7(1.060,2.697)^{*}$ \\
& No & $71(28.4)$ & $179(71.6)$ & 1 & 1 \\
Lengthy admission process & Yes & $36(47.4)$ & $40(52.6)$ & $2.2(1.326,3.700)$ & $2.2(1.279,3.776)^{* *}$ \\
& No & $89(28.9)$ & $219(71.1)$ & 1 & 1 \\
\multirow{2}{*}{ Type of delivery } & SVD & $82(30.0)$ & $191(70.0)$ & 1 & 1 \\
& NSVD & $43(38.7)$ & $68(61.3)$ & $1.5(0.929,2.336)$ & $1.4(0.832,2.206)$ \\
\hline
\end{tabular}

Note: $1=$ reference, $*=$ p-value $<0.05, * *=$ p-value $\leq 0.001$

\section{Discussions}

This study is planned to determine the three phases of delay and its associated factors among labouring mothers. All pregnant women are at risk of obstetric complications. Numerous factors contribute to hinder the utilization of health facility in developing countries at the time of obstetric complication and childbirth. Thaddeus and Maine have categorized the factors as: 1) delay in decision to seek care; 2) delay to arrive at a health facility; and 3) delay to receive adequate care. If these barriers could be overcome, the outcome of the pregnancy would be satisfactory [1].

\subsection{First Delay}

The finding of this investigation in the first delay was that 154 [40.1\%] of mothers had delayed in deciding to seek emergency obstetric care. This is consistent with the study findings in Ethiopia [13]. But inconsistent with the study findings noted in Pakistan/Karachi Civil Hospital, 71\%, in Bangladesh $69.3 \%$, and in Nigeria $57 \%$ [14, 15, 16]. This might be population difference, place and time of study, cultural diversity, health service delivery system, methodological difference, lack of health extension program implementation, and accessibility of primary education for females.

Though the prevalence of this study was lower than other countries study findings, the burden of maternal and newborn mortality and morbidity in our setting which could be attributed to delayed decision in seeking emergency obstetric care is still higher.

Regarding predictors, mother's occupation, husband's education, and number of ANC visits were independently associated with first delay of emergency obstetric care. In this study, unemployed mothers were three times more likely to delay than employed ones. It was similar finding with a study done in Nigeria, and Ethiopia $[13,16]$. This might be dependence on their husbands in the aspect of financial constraints, consequently, which decreases mother's decision making power to seek emergency obstetric care. Mothers who attended greater than four visits of antennal care were [AOR, 0.4; 95\% CI, 0.277, 0.713] 60\% less likely to delay than who attended less than four visits. As antenatal care is one of the pillars of the maternal health services; when a mother had less antenatal contacts, it may have less opportunity to consult care provider on: birth preparedness, decision making about place of delivery and seek early emergency obstetric care.

Similarly, mothers with illiterate husbands were 2.3 times more likely to delay than with literate husbands. This was parallel with study conducted in rural Tanzania, and Ethiopia $[17,18]$. This might be uneducated husband may not perceive the pattern of decision-making power within the family members as key determinants of the place of delivery. Also, the less educated husband's may have poor awareness in delivery care importance; thus affect family member's involvement in decision making. The mean delay time of first delay was three and half hours; this figure is lower than the mean time $(8 \mathrm{hrs}$.) in Amhara region and higher than the study findings in rural Bangladesh in which time required to make a decision to seek care was 72 minutes [13, 15]. Basically, deciding to seek emergency obstetric care should be made before labour initiated i.e. during the birth preparedness but practically we see that all of the mothers decide to seek EmOC after the labour was initiated. This does not augur well for government efforts to reduce delays as a principal point for labouring mothers.

\subsection{Second Delay}

Concerning to second delay, in this study 114 [29.7\%] mothers were encountered transportation problem to reach the health facilities for getting emergency obstetric cares. This finding is coherent with the findings in Ethiopia [31.7\%]; but inconsistent with the study findings in Ghana [43\%] and Pakistan [74\%]; this might be topography difference, expansion and/or closeness of care giving facilities to community, infrastructure improvements, and ambulance facilitation. Even though the finding in this study showed lower figure than others and there is a number of strategies put in place to solve the transportation problem, the magnitude of maternal and newborn morbidity and mortality in our country is still high which could be characterized to delay in reaching to EmOC facility within the recommended one hour.

In this study distance, means of transportation, and mother's education was independently associated with the second delay. Mothers those travelling beyond 5 kilometres from health facilities were fourteen times more likely to delay in reaching to health care facility compared to those 
living within five kilometres. Similarly, the study conducted in Ethiopia, rural Zambia and Pakistan showed independently association of distance with 2 nd delay $[14,19,20]$. The study which conducted on "International Federation Rural Transport Development" inferences that as $10 \%$ increases in distance from a hospital, maternal and neonatal death increased by $2 \%$ [21].

The possible reasons might be: place of residence, road and/or transport inaccessibility, and absence of functioning nearby HF on EmOC services. As health facility too far to reach, the labouring mother might be delayed in getting there and missed life saving EmOC solutions and developed a lifethreatening complication, thus could result in both maternal and newborn morbidity and mortality. A study conducted in rural Zambia showed that facility delivery for a birth within 1 kilometre of a comprehensive health facility are over 10 times higher for a birth whose closest facility is 20 kilometre away or above [19]. Mothers who used other means of transportation were [AOR, 0.6; 95\% CI, 0.314, 0.995] more likely to delays than those used ambulances. It is known that the time between onset of an obstetric emergency and definitive care is critical for the survival of the mother and her baby. According to this study, ambulance was used for means of transportations by 144 [37.5\%] of the mothers, renting car for $150(39.0 \%)$ which including "Bajaj" and the rest $51(13.3 \%)$ and $39(10.2 \%)$ were travelled by private car and on foot/wooden stretcher, respectively. This indicates majority of labouring mothers were not used ambulance services.

Illiterate mothers were 3 folds more likely to delay than literate mothers. This was consistent with findings in Ethiopia [13]. The possible reasons might be education: poor knowledge on birth preparedness and lack of awareness on danger signs and birth complication.

\subsection{Third Delay}

The findings of delay three revealed 125 [32.6\%] of mothers were not got the emergency obstetric care as early as possible upon reaching the healthcare facility. Which was in line with the findings in Ethiopia [30.7\%], but lower than findings in Pakistan (48\%) [13, 14]; the mean delay time was 1.5 hours; this is lower than the mean delay time (4 hours) of Amhara region. This discrepancy could be due to the time gap between these studies, population size, difference in study setting, mother's demographic and socioeconomic characteristics, fee free delivery services implementation, study design, improved in medical logistic supply and professional staff in skilled delivery.

Request multiple referrals, lengthy admission process, and absence of care provider were main predictors of third delay. The mothers who experienced multiple referrals were [AOR, $0.2 ; 95 \%$ CI, 0.068, 0.347] more likely to delay than those having emergency obstetric service at first contact. This was consistent with study done in United Kingdom on a systematic review of the third delay [22]. This might be nonfunctioning health facility in EmOC, lack of basic medical equipment for care giving, absence of skilled delivery service provider or beyond primary health care unit level that needs farther investigation.

Mothers faced lengthy admission processes were 2.2 times higher risk of delaying in receiving EmOC than their cohorts. The possible reasons for this might be lack of awareness the severity of EmOC in some non-professionals such as data clerks, absence of senior professional at labouring mother's arrival, shortage of admission bed and other medical supplies.

Mothers encountered with absence of skilled delivery care providers were 1.7 times more likely to delay compared to their counterparts. This was consistent with done in United Kingdom on a systematic review of the third delay [22]. This might be shortage of skilled man power, staff work load and/or attitude.

In general, the findings showed that the three delays are interlinked; one factor is independently associated to more than one delay. Poor quality of care at the facility level contributes to a perception that adequate care is not available and thus affects the mother's decision about whether or not to seek care. Reaching to healthcare facility may be influenced by income constraints. Therefore, interventions that address quality of care would also help address some of the factors that contribute to delays in the family decision to seek care. Similarly, efforts to shift appropriate care closer to the community would reduce both the transport burden on the family in terms of cost as well as time required to reach care and could affect decision making about where and when to seek care.

\section{Limitations}

First, this study was based at the health facility and the labouring mothers do not represent the general populations of Hadiya zone because only mothers seeking institutional delivery were eligible for the study, making hard to make inferences. Second, labouring mothers were selected based on consecutive sampling strategy which is not based on random selection. Third, midwifes working in the hospital administered the questionnaires and this may have led to social desirability bias. Lastly, the findings may be affected by recall bias since mothers were interviewed soon after birth while mothers physically and emotionally exhausted. Despite these limitations, the study identified a number of barriers that can delay proper utilization of EmOC services among delivery attendees. These barriers can be easily mitigated if properly planned and addressed.

\section{Conclusion and Recommendation}

\subsection{Conclusions}

This study highlights how focuses on the delays for utilizing institutional delivery care. The very high proportion of each delay in this study suggests low access of labouring mothers to health services. Major factors which contributed to delays for utilizing institutional 
delivery were: mother's occupation, distance, mother's education, husband education, number of ANC visits, means of the transportation, lengthy admission process, multiple referrals, and absence of care provider. This study stresses the importance of addressing three delays alongside institutional delivery services to achieve in further reduction of delays. The development of simple, interlinked means to end these three phase hinders should be seen as a priority for future research.

\subsection{Recommendations}

Hospital should be provided with adequate human resources. Strengthen an internal supervision, monitoring, and on-job training activities to avoid lengthy admission process. The hospital should work with their subordinate primary health care provision units to improve a means for mothers' referral system. Educating the health development army (HDA) leaders should be strengthened in order to arrange fee free local transport and to create sense of family's ownership on pregnant mothers' birth preparedness to seek emergency obstetric care. Emphasis should be given for the transportation mechanisms like the ambulance services and orientation should be given for ambulance drivers about risk of delays and its consequence both on mothers and newborn. The health sectors and other responsible bodies should make efforts to increase adult education, income generating mechanism for mothers and encourage women's education to higher levels and improve better access to information for action regarding to early decision to seek EmOC. Finally, we would like to recommend for researchers to conduct community based investigation.

\section{Competing Interests}

The authors declare that they have no competing interests.

\section{Abbreviations}

ANC - Antenatal care, EmOC - Emergency obstetric care, NSVD - None spontaneous vaginal delivery, SVD Spontaneous vaginal delivery

\section{Acknowledgements}

We thank Jimma University for funding this study. But the funding does not cover publication process. Study participants, data collectors, and supervisors are acknowledged for their cooperation during data collection.

\section{References}

[1] Thaddeus S, Maine D. Too far to walk: maternal mortality in context. Social Science \& Medicine. 1994; 38(8): 1091-1100.

[2] Berhan Y, Berhan A. Yifru B, and Asres B, Reasons for persistently high maternal and perinatal mortalities in Ethiopia: Part I, II, and III - Socio-economic and cultural factors. 2014; Special issue 1.

[3] See A. Starrs, "Safe Motherhood Initiative: 20 years and conducting," The Lancet, UN, 2006.

[4] Millennium Project, Investing in Development: A practical plan to achieve the Millennium Development Goals, UNDP, 2005.

[5] UNFPA: Emergency obstetric care: Reducing life-threatening delay. http://www.unfpa.org/public/home/mothers/pid/4385, 2012

[6] Kamara, A. the international federation of gynecology and obstetrics; lessons learned from the PMM network experience. Int J GynecolObstet, 1997. V 59 (Suppl 2) 253-258.

[7] Skilled Attendance at Birth. Available from: http://www.unfpa.org/public/cache/offonce/home/mothers/pid/ 4383 [cited 12 March 2014].

[8] Khan KS, Wojdyla D, Say L, Gülmezoglu AM, Van Look PFA. WHO analysis of causes of maternal death: a systematic review. Lancet 2006; 367: 1066-74.

[9] Hunt P, De Mesquita JB. Reducing maternal mortality: the contribution of the right to the highest attainable standard of health. London: Human Rights Centre, University of Essex and UNFPA, New York, 2010.

[10] UNFPA, Trends in Maternal Health, Challenges in achieving the MDG for maternal mortality In-depth Analysis of the EDHS 2000-2011.

[11] Reuben K. Esena, Mary-Margaret S.: Factors associated with the utilization of skilled delivery services, international journal of scientific \& technology research volume 2 , issue 8 , august 2013 issn 2277-8616.

[12] FMOH: Mini Ethiopian Demographic and Health Survey, 2014.

[13] Worku A. and Kenie S.: Maternal delays in utilizing institutional delivery services, Bahir Dar, Ethiopia Vol. 5, No. 6, 1026-1031 (2013).

[14] Nusrat S., Nazli H, Rizwana S, et al. Socio-demographic Characteristics and the Three Delays of Maternal Mortality: Journal of The College of Physicians and Surgeons Pakistan 2009, Vol. 19 (2): 95-98.

[15] Kaji, T., Keya, Moshiur, R., Ubaidur, R., et al. Barrier of Distance and Transportation Cost to Access Maternity Services in Rural Bangladesh, 2013.

[16] Okusanya BO, Okogbo FO, Momoh MM, et al. Maternal mortality and delay: socio-demographic characteristics of maternal deaths with delay in Irrua, Nigeria. Niger J Med 2007; 16: 38-41.

[17] Mrisho, M, Schellenberg, J. A, Mushi, A. K, Obrist, B., Mshinda, H., Tanner, M., and Schellenberg, D., Factors affecting home delivery in rural Tanzania, 2007.

[18] Daniel B. and Desalegn M. Institutional Delivery Service Utilization and Associated Factors among Child Bearing Age Women in Gobaworeda, Ethiopia. Journal of Gynecology and Obstetrics. Vol. 2, No. 4, 2014, pp. 63-70.

[19] Gabrysch S, Cousens S, Cox J, Campbell OM: The influence of distance and level of care on deliveryplace in rural Zambia, 2011. 
[20] Gaym A. A review of maternal mortality at Jimma Hospital, Southwestern Ethiopia. 2000; 14(2): 215-223.

[21] De Silva R. Transport - The Missing Link? A catalyst for achieving the MDG, Presentation for the International Federation of Rural Transport Development. Retrieved from: http://www.transaid.org/images/
[22] Knight HE, Self A, Kennedy SH: Why Are Women Dying When They Reach Hospital on Time? A Systematic Review of the "Third Delay." (2013) PLoS ONE 8(5): e63846. doi: 10.1371/journal.pone.0063846. 